\title{
INTERSTELLAR SCINTILLATION STUDIES OF PULSARS AND DISTRIBUTION OF SCATTERING PLASMA IN THE LOCAL INTERSTELLAR MEDIUM
}

\author{
N.D. RAMESH BHAT \\ National Astronomy and Ionosphere Center, Arecibo Observatory, U.S.A. \\ YASHWANT GUPTA and A. PRAMESH RAO \\ National Centre for Radio Astrophysics, Pune, India \\ P.B. PREETHI \\ Hindustan College of Engineering, Madras, India
}

\begin{abstract}
Pulsar scintillation measurements from the Ooty Radio Telescope (ORT) are used to investigate the distribution of scattering in the Local Interstellar Medium (LISM; region of $\lesssim 1 \mathrm{kpc}$ of the Sun), specifically the region in and around the Local Bubble. A 3-component model, where the Solar neighborhood is surrounded by a shell of enhanced plasma turbulence, is proposed for the LISM. Further, the Ooty data, along with those from Parkes and other telescopes are used for investigating the distribution of scattering towards the nearby Loop I Superbubble.
\end{abstract}

\section{Introduction}

Interstellar Scintillation (ISS) measurements of pulsars are important inputs for understanding the distribution and the spectrum of radio wave scattering plasma in the Interstellar Medium (ISM). Cordes et al. (1991) and Taylor and Cordes (1993; TC93 hereafter) describe the large-scale distribution of scattering in the Galaxy, wherein the LISM is taken to be more or less uniform. Such a picture is not quite in agreement with our understanding of the LISM from observations at various wavebands. The LISM is known to consist of several large-scale features, most prominent of which is a low-density, X-ray-emitting cavity extending up to a few $100 \mathrm{pc}$ that surrounds the Solar system (cf. Breitschwerdt et al., 1998). This structure, referred to as the Local Bubble, and its environment can potentially influence the dispersion and scintillation properties of nearby pulsars.

\section{Scattering Due to the Local Interstellar Bubble}

From the ISS measurements, we obtain estimates of the line-of-sight-averaged strength of scattering, $\overline{C_{n}^{2}}$, a useful quantity for investigating the nature of distribution of the scattering plasma. There are about two orders of magnitude fluctuations 

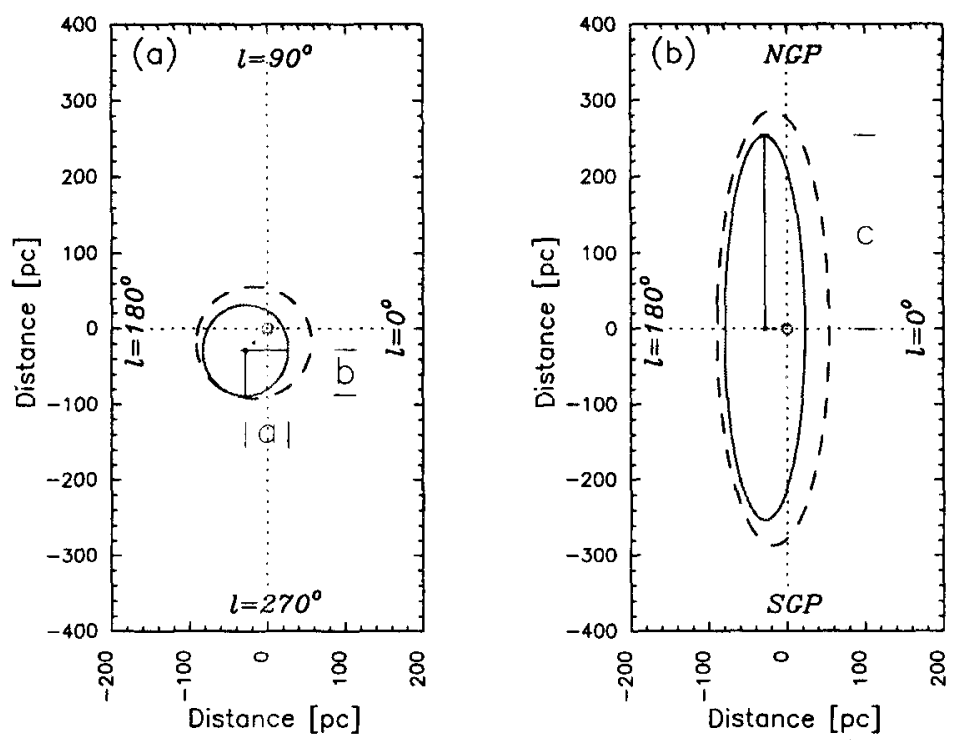

Figure l. Geometry of the local scattering structure which best reproduces the observational results from the Ooty Radio Telescope. Panels (a) and (b) are sections along the Galactic plane and along a plane perpendicular to the Galactic plane and passing through the Galactic poles, respectively.

in $\overline{C_{n}^{2}}$ (comparable to that expected from the TC93 model), and a systematic variation with distance and DM, for nearby $(\lesssim 1 \mathrm{kpc})$ pulsars. Further, observations show several cases of pulsars with comparable DMs and/or at similar distances showing remarkably different scintillation behaviour.

We explain the above results with a 3-component model, where the Solar system resides in a weakly scattering medium, surrounded by a shell of enhanced scattering (see Bhat et al., 1998 for details). The inferred scattering structure has an ellipsoidal morphology and is more extended away from the Galactic plane (Figure 1). The centre is located at $\sim 20-35 \mathrm{pc}$ from the Sun, towards $215^{\circ}<$ $l<240^{\circ}$ and $-20^{\circ}<b<20^{\circ}$. The strength of scattering in the shell material $\left(10^{-0.96}<\int_{0}^{d} C_{n}^{2}(z) d z<10^{-0.55} \mathrm{pc} \mathrm{m}^{-20 / 3}\right)$ is much larger than that in the interior $\left(10^{-4.70}<\overline{C_{n}^{2}}<10^{-4.22} \mathrm{~m}^{-20 / 3}\right)$ and in the ambient ISM $\left(\overline{C_{n}^{2}}<10^{-3.30} \mathrm{~m}^{-20 / 3}\right)$.

The size and morphology inferred for the scattering structure are similar to that of the Local Bubble, as known from X-ray, EUV, UV and optical data. The constraints on the scattering parameters are such that the edge of the bubble can account for much of the enhanced scattering of nearby $(\lesssim 1 \mathrm{kpc})$ pulsars, particularly at higher Galactic latitudes (see Bhat et al., 1998 for details). Interestingly, independent evidence in favor of fairly close-by scattering screens $(\sim 50 \mathrm{pc})$ comes from observations of $\mathrm{cm}$-wave ISS and intra-day variable (IDV) quasars (see e.g. Rickett, this volume). In particular, we note that a screen location $\sim 25 \mathrm{pc}$ and a strength of scattering, $\overline{C_{n}^{2}} \approx 0.2 \mathrm{~m}^{-20 / 3}$ inferred by Dennett-Thorpe and De 
Pulsars and Local ISM

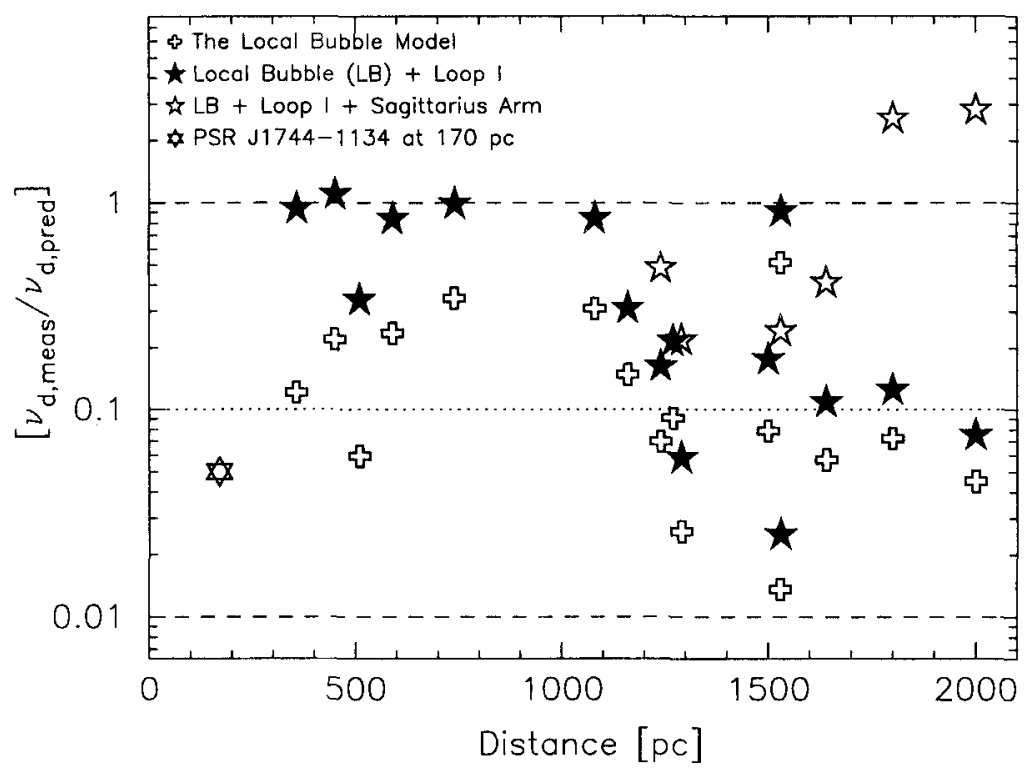

Figure 2. Plots illustrating the enhanced level of scattering of nearby pulsars due to the Local Bubble and the Loop I Superbubble. Ratios of the measured decorrelation bandwidths ( $v_{d, \text { meas }}$ ) to their values as predicted by the models for the distribution of scattering in the Local ISM ( $v_{d}$,pred $)$ are plotted against the distance estimates. The measurements of $v_{d}$ are scaled to $327 \mathrm{MHz}$.

Bruyn (2000; see also this volume) towards the IDV quasar J1819+3845 is in very good agreement with the location of the bubble boundary $(\sim 25-50 \mathrm{pc})$ and the scattering strength expected in this direction from our LISM model.

\section{Scattering Towards the Loop I Superbubble}

Our understanding of the structure of the LISM has considerably improved over recent years (cf. Breitschwerdt et al., 1998). Observations suggest that there are several other bubbles in the LISM. The nearest neighbor to the Local Bubble may be the Loop I Superbubble (Egger, 1998). With a size $\sim 300 \mathrm{pc}$ and located at $\sim 170 \mathrm{pc}$ towards $l \sim 330^{\circ}, b \sim 20^{\circ}$, Loop I covers a large region of the sky, hence can potentially influence the dispersion and scintillation of nearby pulsars. A critical comparison study of recent scintillation measurements (Johnston et al., 1998; Bhat et al., 1999; Cordes, 1986) of pulsars whose lines-of-sight (LOSs) intersect Loop I with their predictions from the Local Bubble model reveal large discrepancies, whereby the level of enhanced scattering is much higher than expected (Figure 2).

We have extended the LISM model by incorporating the scattering due to turbulent plasma possibly associated with Loop I. PSR J1744-1134 and PSR J1456- 
6843 turned out to be very promising for constraining the scattering due to the Loop I boundary. Toscano et al. (1999) report precise estimates of parallax distance for PSR J1744-1134. With the new distance estimate ( $357 \pm 35 \mathrm{pc})$, the second boundary of Loop I is located midway along the line-of-sight, hence can potentially account for much of its enhanced scattering. Such enhanced scattering could not have been explained if the pulsar was to be located at $170 \mathrm{pc}$ (indicated by unfilled star in Figure 2), as suggested by the TC93 model. The improved model accounts for the scattering of most pulsars within $\sim 1 \mathrm{kpc}$. For more distant pulsars, the discrepancy is not completely removed. A closer examination of their LOSs suggests the Sagittarius spiral arm to be most plausible source of enhanced scattering. The discrepancies are further reduced on incorporating the scattering due to this spiral arm (Figure 2).

\section{Acknowledgements}

NDRB and YG acknowledge the International Astronomical Union and the National Natural Science Foundation for support with travel grants.

\section{References}

Bhat, N.D.R., Gupta, Y. and Rao, A.P.: 1998, Astrophys. J. 500, 262.

Bhat, N.D.R., Rao, A.P. and Gupta, Y.: 1999, Astrophys. J. Suppl. 121, 483.

Breitschwerdt, D., Freyberg, M.J. and Trümper, J.: 1998, Proc. IAU Coll. 166.

Cordes, J.M.: 1986, Astrophys. J. 311, 183.

Cordes, J.M., Weisberg, J.M., Frail, D.A., et al.: 1991, Nature 354, 121.

Dennett-Thorpe, J. and de Bruyn, A.G.: 2000, Astrophys. J. Lett. 529, 65.

Egger, R.: 1998, Lecture Notes in Physics 506, 287.

Johnston, S., Nicastro, L. and Koribalski, B.: 19989, Mon. Not. R. Astron. Soc. 297, 108.

Taylor, J.H. and Cordes, J.M.: 1993, Astrophys. J. 411, 674.

Toscano, M., Britton, M.C., Manchester, R.N., et al:: 1999, Astrophys. J. 523, L171. 